IU-MSTP $/ 35$

hep-lat/9906015

June 1999

\title{
Non-commutative Differential Calculus and the Axial Anomaly in Abelian Lattice Gauge Theories
}

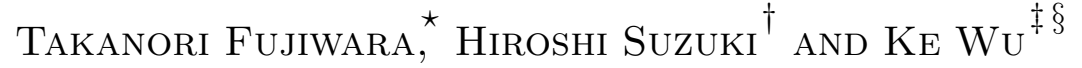 \\ Department of Mathematical Sciences, Ibaraki University, Mito 310-8512, Japan
}

\begin{abstract}
The axial anomaly in lattice gauge theories has a topological nature when the Dirac operator satisfies the Ginsparg-Wilson relation. We study the axial anomaly in Abelian gauge theories on an infinite hypercubic lattice by utilizing cohomological arguments. The crucial tool in our approach is the non-commutative differential calculus (NCDC) which makes the Leibniz rule of exterior derivatives valid on the lattice. The topological nature of the "Chern character" on the lattice becomes manifest in the context of NCDC. Our result provides an algebraic proof of Lüscher's theorem for a four-dimensional lattice and its generalization to arbitrary dimensions.
\end{abstract}

PACS numbers: 11.15.Ha, 11.30.Rd, 02.40.-k

Keywords: lattice gauge theory, axial anomaly, noncommutative geometry

\footnotetext{
* E-mail: fujiwara@mito.ipc.ibaraki.ac.jp

† E-mail: hsuzuki@mito.ipc.ibaraki.ac.jp

$\ddagger$ On leave of absence from Institute of Theoretical Physics, Academia Sinica, P. O. Box 2735, Beijing 100080, China.

$\S$ E-mail: wuke@mito.ipc.ibaraki.ac.jp
} 


\section{Introduction}

The non-perturbative formulation of chiral gauge theories is a long standing problem in theoretical particle physics. It appears that, if one wishes to regularize chiral gauge theories by keeping the gauge symmetry manifest, one has to first understand the structure of gauge anomalies defined in a theory with a finite ultraviolet cutoff. Therefore it is quite important to study the axial (gauge) anomalies on the lattice while keeping the lattice spacing finite.

Recently, there have been remarkable developments regarding this problem. First, gauge covariant local Dirac operators which satisfy the Ginsparg-Wilson (GW) relation [1] were discovered $[2,3,4]$. It was soon recognized that GW relation implies the index theorem [3,5] and the exact chiral symmetry [5] of the fermion action. The chiral symmetry is however anomalous due to the non-trivial Jacobian factor and the Jacobian is related to the index, as is in the continuum theory [6]. The Jacobian associated with the local chiral transformation, i.e., the chiral or axial anomaly, is given by the GW Dirac operator $D$ as "

$$
q(x)=\operatorname{tr} \gamma_{5}\left[\delta_{x, x}-\frac{1}{2} D(x, x)\right]
$$

where tr stands for the trace over the spinor indices. The perturbative evaluation of the quantity $q(x)$ in the continuum limit was carried out in Ref. [7] by using the overlap-Dirac operator [4] and it was confirmed that the field $q(x)$ reproduces the anomaly in the continuum theory. See also Ref. [8]. It can even be shown [9] that the continuum limit of Eq. (1.1) in general coincides with the axial anomaly in the continuum theory, if the Dirac operator behaves properly in this limit. This issue is addressed also in Ref. [10].

The index theorem $[3,5]$ states that the volume integral of the axial anomaly $q(x)$ is integer valued. This would imply that the volume integral of $q(x)$ is invariant

I Throughout this article, the lattice spacing is taken to be unity $a=1$. 
under a local variation of the gauge field*

$$
\sum_{x} \delta q(x)=0
$$

Solely from this topological property combined with the gauge invariance and the locality of the field $q(x)$, it was shown [11] that, in Abelian gauge theories on a fourdimensional infinite hypercubic lattice, the field $q(x)$ has the following structure:

$$
q(x)=\alpha+\beta_{\mu \nu} F_{\mu \nu}(x)+\gamma \varepsilon_{\mu \nu \rho \sigma} F_{\mu \nu}(x) F_{\rho \sigma}(x+\widehat{\mu}+\widehat{\nu})+\partial_{\mu}^{*} k_{\mu}(x),
$$

where $\alpha, \beta_{\mu \nu}$ and $\gamma$ are constants and $\varepsilon_{\mu \nu \rho \sigma}$ is the Levi-Civita symbol; $F_{\mu \nu}(x)$ is the Abelian field strength and $\widehat{\mu}$ stands for the unit vector in direction $\mu$. In the last term, the current $k_{\mu}(x)$ is a gauge invariant quantity that locally depends on the gauge field; the symbol $\partial_{\mu}^{*}$ stands for the backward difference operator. In the next section, we summarize our notation and a set of assumptions under which Eq. (1.3) holds.

In Eq. (1.3), the gauge invariance of the current $k_{\mu}(x)$ distinguishes the last term from other terms which may also be written as total divergences (see below) but only in terms of gauge variant currents. Equation (1.3) is important because it tells us the structure of the axial anomaly with a finite lattice spacing. (The first two terms are absent for the axial anomaly in four dimensions, because in this case $q(x)$ behaves as pseudo-scalar quantity under the lattice symmetries.) Roughly speaking, the current $k_{\mu}(x)$ represents a lattice artifact in the axial anomaly and it may be removed by a gauge invariant local redefinition of the axial current. By

* This topological property of the anomaly can be shown even on an infinite lattice, for which notion of the index might be ill-defined. Introduce the operator $\Gamma_{5}=\gamma_{5}(1-D)$. Then GW relation $\gamma_{5} D+D \gamma_{5}=D \gamma_{5} D$ implies $\Gamma_{5}^{2}=1$ and $\left\{\Gamma_{5}, \gamma_{5} \delta D\right\}=0$. ¿From these, we have $\operatorname{Tr} \gamma_{5} \delta D=\operatorname{Tr} \gamma_{5} \delta D \Gamma_{5}^{2}=\operatorname{Tr} \Gamma_{5} \gamma_{5} \delta D \Gamma_{5}=--\operatorname{Tr} \gamma_{5} \delta D=0$, here $\operatorname{Tr}$ stands for $x$ integration of the diagonal $(x x)$ components as well as the spinor trace. This is equivalent to $\sum_{x} \delta q(x)=0$. Note that the sum $\operatorname{Tr} \gamma_{5} \delta D$ is well-defined even on the infinite lattice, at least if the Dirac operator $D$ has a local dependence on the gauge potential and if the variation of the gauge potential is well-localized. 
defining chiral properties with respect to the GW chiral matrix [12], the (covariant) gauge anomaly in chiral gauge theories is also given by the field $q(x)$. Therefore, the theorem (1.3) also specifies the structure of the Abelian gauge anomaly with a finite lattice spacing. The coefficient $\gamma$ is given by the coefficient of the anomaly in the continuum theory. ${ }^{* *}$ These facts suggest that, when the anomaly cancellation condition in the continuum theory is fulfilled, i.e., when $\gamma=0$, a gauge invariant lattice formulation of Abelian chiral gauge theories is possible, by removing the artificial breaking of the gauge symmetry on the lattice, i.e., the current $k_{\mu}(x)$. In the meantime, Neuberger [13] pointed out that a similar "anomaly cancellation" mechanism works with the overlap formalism [14], at least for a particular kind of gauge field configurations. Finally, on the basis of the observation (1.3), Lüscher [15] gave an existence proof of a gauge invariant lattice formulation of Abelian chiral gauge theories, which is consistent with other physical requirements. See Ref. [16] for recent related works.

In this paper, we present an alternative proof of the theorem (1.3) with the aim of a more transparent understanding of it. The idea is the following. The statements (1.2) and (1.3) have the counterpart in the continuum field theory, which has been proven $[17,18]$ by utilizing algebraic techniques introduced in Ref. [19]. It is thus natural to expect similar algebraic techniques may be applied to the present problem in the lattice gauge theory. However, as is well-known, the Leibniz rule does not hold for differences on a lattice and this has been an obstruction to such an algebraic approach. Here we avoid this difficulty related to the Leibniz rule by introducing the notion of non-commutative differential calculus (NCDC) on discrete set and groups [20-23]. Some applications of this notion to lattice (field) theories have been discussed in Refs. [22,23]. See also Ref. [24]. By using NCDC, we may apply the almost identical cohomological arguments as in the continuum

** This may be seen as follows: From the dimensional analysis, the coefficient $\gamma$ cannot depend on the spacing $a$. On the other hand, if the GW-Dirac operator $D$ behaves properly in the limit $a \rightarrow 0$, it can be shown [9] that $\lim _{a \rightarrow 0} q(x)=\gamma^{\prime} \varepsilon_{\mu \nu \rho \sigma} F_{\mu \nu}(x) F_{\rho \sigma}(x)$ reproduces the anomaly in the continuum theory. A comparison with Eq. (1.3) in the limit $a \rightarrow 0$ shows $\gamma=\gamma^{\prime}$. 
theory, such as the descent equation [19], to the present problem. As an extra bonus of this algebraic approach, we can not only prove Eq. (1.3) but also easily obtain its generalization to arbitrary dimensions, because the algebra involved is independent of the dimensionality of the lattice.

Here we are not claiming that a certain non-commutativity has to be implemented on the lattice. We use NCDC simply as a convenient tool in intermediate steps of extracting relations which are valid on a lattice even in the conventional sense. It must be possible to show the resulting relations without relying on NCDC; this is actually the case as is briefly discussed in Ref. [25].

In a recent interesting paper [26], it was pointed out that a solution to Eq. (1.2) in (4+2)-dimensional non-Abelian gauge theories (here 4-dimensions are discretized and 2-dimensions are continuous) is directly related to a gauge invariant lattice formulation of non-Abelian chiral gauge theories in 4-dimensions. We expect that algebraic approaches similar to the present one should be useful to study such problems also in non-Abelian theories. This is our another motivation of the present study and the present paper may be regarded as a first step toward this direction.

The organization of this paper is as follows. In the next section, we present some preparation including a set of basic assumptions, a brief review of NCDC and a quick introduction of the BRST transformation. In Sec. 3, we state our main result which generalizes Eq. (1.3) to arbitrary dimensions. To prove our main theorem, we need several lemmas, and each of these lemmas has the counterpart in the continuum field theory $[17,18]$. In Sec. 4, we give the proof of these lemmas. Section 5 is devoted to concluding remarks. 


\section{Preparation}

\subsection{BASIC ASSUMPTIONS}

Throughout this paper, we consider a $D$-dimensional hypercubic regular lattice with an infinite extent. The lattice spacing is taken to be unity $a=1$. We define the forward difference operator $\partial_{\mu}$ and the backward difference operator $\partial_{\mu}^{*}$ as

$$
\begin{aligned}
& \partial_{\mu} f(x)=f(x+\widehat{\mu})-f(x), \\
& \partial_{\mu}^{*} f(x)=f(x)-f(x-\widehat{\mu}),
\end{aligned}
$$

where $f(x)$ is an arbitrary function on the lattice and $\widehat{\mu}$ stands for the unit vector in direction $\mu$. We also introduce the Abelian field strength $F_{\mu \nu}(x)$ by

$$
F_{\mu \nu}(x)=\frac{1}{i} \ln U_{\mu}(x) U_{\nu}(x+\widehat{\mu}) U_{\mu}(x+\widehat{\nu})^{-1} U_{\nu}(x)^{-1},
$$

where $U_{\mu}(x) \in U(1)$ is the link variable on the link that connects the points $x$ and $x+\widehat{\mu}$. We always assume the "admissibility" of the gauge field configuration [11]:

$$
\sup _{x, \mu, \nu}\left|F_{\mu \nu}(x)\right|<\epsilon
$$

for a fixed constant $0<\epsilon<\pi / 3$. Under this condition, one can associate (up to integer valued gauge transformations) the gauge potential $A_{\mu}(x)$ with the link variable as $U_{\mu}(x)=e^{i A_{\mu}(x)}$, and the field strength is given by $F_{\mu \nu}(x)=\partial_{\mu} A_{\nu}(x)-$ $\partial_{\nu} A_{\mu}(x)$ [11]. In particular, the Bianchi identity $\partial_{[\mu} F_{\nu \rho]}(x)=0$ holds under the condition (2.3).

Let $f(x)$ be a functional of the external gauge potential $A_{\mu}$. We say that $f(x)$ locally depends on the gauge potential $A_{\mu}$ if the dependence of $f(x)$ on the gauge potential at distant points is at least exponentially weak. We say that $f(x)$ smoothly depends on the gauge potential $A_{\mu}$ if the dependence of $f(x)$ is sufficiently smooth so that an arbitrary number of derivatives with respect to $A_{\mu}$ 
around $A_{\mu}=0$ may be considered. To prove Eq. (1.3) and its generalization, the axial anomaly $q(x)(1.1)$ must locally and smoothly depend on the external gauge potential. From Eq. (1.1), this is equivalent to the requirement that so is the Dirac operator $D(x, x)$. Although a Dirac operator which satisfies GW relation cannot be ultralocal in general [27], i.e., the dependence cannot be restricted within any finite lattice distance, the dependence on distant points is exponentially weak [28] at least for the overlap-Dirac operator [4]; we assume this holds in general. The necessary smoothness of the dependence is also ensured by the overlap-Dirac operator $[28,11,15]$; we assume this also holds in general.

\subsection{Non-COMmutative DifFERENTIAL CALCUlus}

Here we introduce the differential calculus on the lattice. See also Ref. [11]. The bases of the 1-form on a $D$-dimensional hypercubic lattice are defined as abstract objects which satisfy the Grassmann algebra:

$$
d x_{1}, d x_{2}, \cdots, d x_{D}, \quad d x_{\mu} d x_{\nu}=-d x_{\nu} d x_{\mu}
$$

By using these bases, a generic $n$-form is defined by

$$
f(x)=\frac{1}{n !} f_{\mu_{1} \cdots \mu_{n}}(x) d x_{\mu_{1}} \cdots d x_{\mu_{n}}
$$

where the summation of repeated indices is understood. The exterior derivative (or difference more precisely) is defined by the forward difference operator in Eq. (2.1) as

$$
d f(x)=\frac{1}{n !} \partial_{\mu} f_{\mu_{1} \cdots \mu_{n}}(x) d x_{\mu} d x_{\mu_{1}} \cdots d x_{\mu_{n}} .
$$

This definition immediately implies the nilpotency of the exterior derivative $d^{2}=0$. We also introduce the gauge potential 1-form and the field strength 2 -form by

$$
\begin{aligned}
& A(x)=A_{\mu}(x) d x_{\mu} \\
& F(x)=\frac{1}{2} F_{\mu \nu}(x) d x_{\mu} d x_{\nu}=\frac{1}{2}\left[\partial_{\mu} A_{\nu}(x)-\partial_{\nu} A_{\mu}(x)\right] d x_{\mu} d x_{\nu}=d A(x) .
\end{aligned}
$$

Note that the Bianchi identity $d F(x)=0$ holds, once the field strength is expressed 
by the gauge potential in this way.

The essence of NCDC $[20,21]$ on an infinite lattice $[22,23]$ is the following relation:

$$
d x_{\mu} f(x)=f(x+\widehat{\mu}) d x_{\mu}
$$

Namely a function on the lattice and the basis of 1-form do not simply commute; rather the argument of the function is shifted along $\mu$-direction by one unit when commuting these two objects. The remarkable fact, which follows from the noncommutativity (2.8), is that the Leibniz rule of the exterior derivative $d$ holds. With Eqs. (2.6), (2.1) and (2.8), one can easily confirm that

$$
d[f(x) g(x)]=d f(x) g(x)+(-1)^{n} f(x) d g(x),
$$

for arbitrary forms $f(x)$ and $g(x)$ (here $f(x)$ is an $n$-form). The validity of this Leibniz rule is crucial in our approach. We emphasize again that the exterior derivative $d$ is defined with the difference operator $\partial_{\mu}$ in Eq. (2.1).

\subsection{AbELIAN BRST transformation}

In this subsection, we quickly introduce the BRST transformation and the associated differential form in the BRST superspace [19]. It is possible to study the present problem in Abelian gauge theories without introducing such a sophisticated machinery [25]. However, we think that formulating the problem in this way would be useful to generalize the present approach to non-Abelian cases. The Abelian BRST transformation $\delta_{B}$ is defined as usual by (but note that $\partial_{\mu}$ is the difference operator)

$$
\delta_{B} A_{\mu}(x)=\partial_{\mu} c(x), \quad \delta_{B} c(x)=0
$$

where $c(x)$ is the Faddeev-Popov ghost associated with the Abelian gauge transformation. Note that the field strength is $\operatorname{BRST}$ invariant $\delta_{B} F_{\mu \nu}(x)=0$ and 
the BRST transformation is nilpotent $\delta_{B}^{2}=0$. We may also introduce the antighost $\bar{c}(x)$ and the Nakanishi-Lautrup field $B(x)$, and set $\delta_{B} \bar{c}(x)=i B(x)$ and $\delta_{B} B(x)=0$. However these fields play no role in the following analyses. Next we introduce the Grassmann coordinate $\theta$ and define the BRST exterior derivative by

$$
s=\delta_{B} \cdot d \theta
$$

Note that the usual 1-form $d x_{\mu}$ and the BRST 1-form $d \theta$ anti-commute each other $d x_{\mu} d \theta=-d \theta d x_{\mu}$ and the BRST 1-form $d \theta$ commutes with itself $d \theta d \theta \neq 0$. Therefore, for a Grassmann-even(-odd) $n$-form $f(x)$, we have

$$
d \theta f(x)= \pm(-1)^{n} f(x) d \theta .
$$

Also we have

$$
s^{2}=\{s, d\}=0
$$

where the first relation follows from $\delta_{B}^{2}=0$. Finally, we introduce the ghost 1 -form by

$$
C(x)=c(x) d \theta
$$

In terms of these forms, the BRST transformation (2.10) is expressed as

$$
s A(x)=-d C(x), \quad s C(x)=0 .
$$

Of course we have $s F(x)=0$. Note that $c(x)^{2}=C(x)^{2}=0$ in Abelian cases. 


\section{The main result}

We can now state our main result:

Theorem. Let $q(x)$ be a gauge invariant scalar field on a $D$-dimensional infinite hypercubic lattice. Suppose that the field $q(x)$ is locally and smoothly depending on the Abelian gauge potential $A_{\mu}$ and that $q(x)$ is topological, namely,

$$
\sum_{x} \delta q(x)=0
$$

under an arbitrary local variation of the gauge potential $A_{\mu}$. Then $q(x)$ has the following structure:

$$
\begin{aligned}
q(x)= & \alpha+\sum_{n=1}^{[D / 2]} \beta_{\mu_{1} \nu_{1} \mu_{2} \nu_{2} \cdots \mu_{n} \nu_{n}} F_{\mu_{1} \nu_{1}}(x) F_{\mu_{2} \nu_{2}}\left(x+\widehat{\mu}_{1}+\widehat{\nu}_{1}\right) \cdots \\
& \times F_{\mu_{n} \nu_{n}}\left(x+\widehat{\mu}_{1}+\widehat{\nu}_{1}+\cdots+\widehat{\mu}_{n-1}+\widehat{\nu}_{n-1}\right) \\
& +\partial_{\mu}^{*} k_{\mu}(x),
\end{aligned}
$$

where $\alpha$ and $\beta_{\mu_{1} \nu_{1} \cdots \mu_{n} \nu_{n}}$ are constants which are totally anti-symmetric in indices; in particular, $\beta_{\mu_{1} \nu_{1} \cdots \mu_{D / 2} \nu_{D / 2}}$ is a constant multiple of the D-dimensional LeviCivita symbol. The current $k_{\mu}(x)$ is gauge invariant and is locally and smoothly depending on the gauge potential $A_{\mu}$ and $\left.k_{\mu}(x)\right|_{A=0}=0$.

The part $F F \cdots F$ of Eq. (3.2) corresponds to the Chern character in the continuum theory [19] and the gauge invariant local current $k_{\mu}(x)$ may be regarded as a lattice artifact in the anomaly. Equation (3.2) reproduces Eq. (1.3) for $D=4$ $\left(\beta_{\mu \nu \rho \sigma}=\gamma \varepsilon_{\mu \nu \rho \sigma}\right)$ and provides its higher-dimensional generalization. The coordinate dependences in the "Chern character" $F F \cdots F$ in Eq. (3.2) can naturally be understood in the framework of NCDC, as we will see shortly. In particular, in terms of NCDC, it is quite easy to see that $F F \cdots F$ is in fact a total divergence on the lattice and it has the topological property (3.1). 
The main theorem (3.2) can be shown straightforwardly, once the following four lemmas are established. The first two lemmas are concerning the "De Rham cohomology" on the infinite lattice. We simply quote the first one from Ref. [11]:

Poincaré lemma. Let $\eta(x)$ be a $p$-form that decreases at least exponentially at infinity. If it is $d$-closed $d \eta(x)=0$ for $p<D$ or $\sum_{x} \eta(x)=0$ for $p=D$, then one can construct a $(p-1)$-form $\chi(x)$ such that

$$
\eta(x)=d \chi(x) .
$$

The form $\chi(x)$ also decreases at least exponentially at infinity.

The construction [11] of the form $\chi(x)$ from the original form $\eta(x)$ in Eq. (3.3) requires a certain reference point other than the point $x$. If one naively applies the construction to a certain field $\eta(x)$, with a particular choice of the reference point (e.g., the origin), then the translational invariance is broken and the resulting field $\chi(x)$ does not locally depend on the external gauge potential even if the original field $\eta(x)$ locally depends on the gauge potential. ${ }^{\dagger}$ Thus, in the proof of Eq. (1.3) in Ref. [11], the construction is always applied to "bi-local" fields such as $\eta(x, y)$, by taking $y$ or $x$ as the reference point. This complication associated to the reference point may however be avoided as follows:

Algebraic Poincaré lemma. Let $\eta(x)$ be a $p$-form that is locally and smoothly depending on the gauge potential $A_{\mu}$ and $\left.\eta(x)\right|_{A=0}=0$. If it is $d$-closed $d \eta(x)=0$ for $p<D$ or $\sum_{x} \eta(x)=0$ for $p=D$, then there exists a $(p-1)$-form $\chi(x)$ such that

$$
\eta(x)=d \chi(x) .
$$

Moreover the form $\chi(x)$ is also locally and smoothly depending on the gauge potential $A_{\mu}$ and $\left.\chi(x)\right|_{A=0}=0$.

* The Poincaré lemma for 1-forms on a two-dimensional lattice has been proven in Ref. [29] in the context of NCDC.

$\dagger$ This point was overlooked in the first version of this paper. We are grateful to M. Lüscher for calling this point to our attention. 
The above lemma (3.4) is a simple consequence of the original Poincaré lemma (3.3), as we will see in the next section. Nevertheless it requires no explicit reference point and its use considerably simplifies the following arguments; the algebraic Poincaré lemma is rather handy.

The next one is concerning the BRST cohomology in Abelian gauge theories:

Abelian BRST cohomology. Let $X(x)$ be a form that is locally and smoothly depending on the gauge potential $A_{\mu}$ and possibly on the ghost field c. Suppose that the form $X(x)$ has a definite (non-negative) ghost number. Then there exist the forms $\Omega(x)$ and $Y(x)$ such that

$s X(x)=0 \Leftrightarrow X(x)= \begin{cases}\Omega(x), & \text { for } X \text { with the ghost number zero, } \\ C(x) \Omega(x)+s Y(x), & \text { for } X \text { with the ghost number one, } \\ s Y(x), & \text { otherwise, }\end{cases}$

where the form $\Omega(x)$ depends on the gauge potential $A_{\mu}$ and it is gauge invariant; the form $Y(x)$ may depend on the ghost field $c$ as well as on the gauge potential. These dependences are local and smooth.

The next lemma is the crucial one for the proof of the theorem:

Abelian covariant Poincaré lemma. Let $\omega_{p}(x)$ be a gauge invariant $p$-form that is locally and smoothly depending on the gauge potential $A_{\mu}$ and $\left.\omega_{p}(x)\right|_{A=0}=$ 0 . If it is $d$-closed $d \omega_{p}(x)=0$ for $p<D$ or if it is d-exact $\omega_{p}(x)=d \chi_{p-1}(x)$ for $p=D$ with the $(p-1)$-form $\chi_{p-1}(x)$ that is locally and smoothly depending on the gauge potential and $\left.\chi_{p-1}(x)\right|_{A=0}=0$, then there exists a gauge invariant $(p-1)$-form $\eta(x)$ such that

$$
\omega_{p}(x)=P(F(x))+d \eta(x)
$$

where $P(F)$ is a polynomial of the field strength 2-form $F$ with $P(0)=0$; all the coefficients of the polynomial are placed on the right of $F$ 's as $P(F)=F^{n} B+\cdots$. 
The form $\eta(x)$ is locally and smoothly depending on the gauge potential $A_{\mu}$ and $\left.\eta(x)\right|_{A=0}=0$.

The proof of the above lemmas (except the first one, for which we refer to Ref. [11]) will be given in the next section. Here we show how the main theorem (3.2) follows from these lemmas. (In fact, we need the Poincaré lemma (3.3) and the Abelian covariant Poincaré lemma (3.6) in the proof of the theorem. The other two, the algebraic Poincaré lemma and the Abelian BRST cohomology are used to show the Abelian covariant Poincaré lemma.) First, as noted in Ref. [11], an arbitrary smooth functional of the gauge potential $A_{\mu}$ can be represented as

$$
q(x)=\alpha+\sum_{y} A_{\nu}(y) j_{\nu, y}(x)
$$

where $\alpha=\left.q(x)\right|_{A=0}$ and the quantity $j_{\nu, y}(x)$ has been introduced by ${ }^{\star}$

$$
j_{\nu, y}(x)=\int_{0}^{1} d t\left[\frac{\partial q(x)}{\partial A_{\nu}(y)}\right]_{A \rightarrow t A} .
$$

We then introduce the $D$-form $J_{\nu, y}(x)$ by multiplying the volume form $d^{D} x=$ $d x_{1} \cdots d x_{D}$ to $j_{\nu, y}(x), J_{\nu, y}(x)=j_{\nu, y}(x) d^{D} x$. The $x$-integration of the $D$-form $J_{\nu, y}(x)$ however vanishes due to the topological property of the field $q(x)$, Eq. (3.1):

$$
\sum_{x} J_{\nu, y}(x)=\int_{0}^{1} d t\left[\sum_{x} \frac{\partial q(x)}{\partial A_{\nu}(y)}\right]_{A \rightarrow t A} d^{D} x=0 .
$$

Also the assumed locality property of $q(x)$ implies that $j_{\nu, y}(x)(3.8)$ and $J_{\nu, y}(x)$ decrease at least exponentially as $|x-y| \rightarrow \infty$. We can thus apply the original

\footnotetext{
$\star$ The substitution $A_{\mu} \rightarrow t A_{\mu}$ with $0 \leq t \leq 1$ preserves the admissibility (2.3) and thus preserves the locality and the smoothness of the Dirac operator [11].
} 
Poincaré lemma (3.3) to the $D$-form $J_{\nu, y}(x)$ to yield

$$
J_{\nu, y}(x)=d T_{\nu, y}(x)
$$

The important point to note here that the construction [11] of the $(D-1)$ form $T_{\nu, y}(x)$ guarantees that, by taking the point $y$ as the reference point, $T_{\nu, y}(x)$ also possesses the same locality property as $J_{\nu, y}(x)$. The smoothness is also preserved by the construction. We notice that the construction in Ref. [11] works even with NCDC. Therefore, from Eqs. (3.7) and (3.10), we have

$$
\begin{aligned}
q(x) d^{D} x & =\alpha d^{D} x+d \sum_{y} A_{\nu}(y) T_{\nu, y}(x) \\
& =\alpha d^{D} x+d \chi(x) .
\end{aligned}
$$

Due to the above locality property of $T_{\nu, y}(x)$, the summation over $y$ in the second term is well-convergent and we see that the $(D-1)$-form $\chi(x)$ is locally and smoothly depending on the gauge potential. We also note that the $D$-form $d \chi(x)$ is gauge invariant because $q(x)$ is gauge invariant by assumption and $\left.\chi(x)\right|_{A=0}=0$. From these facts, we see that the requisites of the Abelian covariant Poincaré lemma (3.6) are fulfilled for the $d \chi$-part of Eq. (3.11) and thus

$$
q(x) d^{D} x=\alpha d^{D} x+P(F(x))+d \eta(x) .
$$

The lemma (3.6) also says that the $(D-1)$-form $\eta(x)$ is gauge invariant and is locally and smoothly depending on the gauge potential $A_{\mu}$ and $\left.\eta(x)\right|_{A=0}=0$.

To extract the information on the field $q(x)$ from Eq. (3.12), we have to factor out the volume form $d^{D} x$ from both sides of the equation. For the monomial of 
the field strength 2-form, this operation yields

$$
\begin{array}{r}
F(x)^{n} B=\frac{1}{2} F_{\mu_{1} \nu_{1}}(x) d x_{\mu_{1}} d x_{\nu_{1}} \frac{1}{2} F_{\mu_{2} \nu_{2}}(x) d x_{\mu_{2}} d x_{\nu_{2}} \cdots \frac{1}{2} F_{\mu_{n} \nu_{n}}(x) d x_{\mu_{n}} d x_{\nu_{n}} \\
\times \frac{1}{(D-2 n) !} B_{\rho_{1} \cdots \rho_{D-2 n}} d x_{\rho_{1}} \cdots d x_{\rho_{D-2 n}} \\
=\frac{1}{2^{n}(D-2 n) !} \varepsilon_{\mu_{1} \nu_{1} \mu_{2} \nu_{2} \cdots \mu_{n} \nu_{n} \rho_{1} \cdots \rho_{D-2 n}} B_{\rho_{1} \cdots \rho_{D-2 n}} \\
\times F_{\mu_{1} \nu_{1}}(x) F_{\mu_{2} \nu_{2}}\left(x+\widehat{\mu}_{1}+\widehat{\nu}_{1}\right) \cdots \\
\times F_{\mu_{n} \nu_{n}}\left(x+\widehat{\mu}_{1}+\widehat{\nu}_{1}+\cdots+\widehat{\mu}_{n-1}+\widehat{\nu}_{n-1}\right) d^{D} x
\end{array}
$$

where $\varepsilon_{\mu_{1} \cdots \mu_{D}}$ is the $D$-dimensional Levi-Civita symbol (we define $\varepsilon_{12 \cdots D}=1$ ). In deriving the last expression, we have used the basic property of NCDC, Eq. (2.8). By defining $\beta$ as the dual of $B$, we thus obtain the $F F \cdots F$ part of Eq. (3.2). Note that the coordinate dependences of the "Chern character" have been easily obtained by the repeated applications of the non-commutative rule (2.8). Finally the $d$-exact piece of Eq. (3.12), $d \eta(x)$, can be expressed as a total divergence of the dual vector $k_{\mu}(x), k_{\mu}(x)=\varepsilon_{\mu \nu_{1} \cdots \nu_{D-1}} \eta_{\nu_{1} \cdots \nu_{D-1}}(x+\widehat{\mu}) /(D-1)$ !, times the volume form $d^{D} x$. This completes the proof of the theorem (3.2).

In the context of NCDC, the topological nature of the Chern character is also manifest because we can use the Leibniz rule. For example, we have

$$
\begin{aligned}
F(x)^{D / 2}= & d\left[A(x) F(x)^{D / 2-1}\right] \\
= & \frac{1}{2^{D / 2-1}} \varepsilon_{\mu_{1} \nu_{1} \mu_{2} \nu_{2} \cdots \mu_{D / 2} \nu_{D / 2}} \\
& \quad \times \partial_{\mu_{1}}\left[A_{\nu_{1}}(x) F_{\mu_{2} \nu_{2}}\left(x+\widehat{\nu}_{1}\right) F_{\mu_{3} \nu_{3}}\left(x+\widehat{\nu}_{1}+\widehat{\mu}_{2}+\widehat{\nu}_{2}\right) \cdots\right. \\
& \left.\quad \times F_{\mu_{D / 2} \nu_{D / 2}}\left(x+\widehat{\nu}_{1}+\widehat{\mu}_{2}+\widehat{\nu}_{2}+\cdots+\widehat{\mu}_{D / 2-1}+\widehat{\nu}_{D / 2-1}\right)\right] d^{D} x
\end{aligned}
$$

where uses of the Bianchi identity $d F(x)=0$ and the basic property of NCDC (2.8) have been made. In Eqs. (3.13) and (3.14), we must use the non-commutative rule (2.8), because the lemma (3.6) holds only in terms of NCDC, as we will see in the next section. In other words, Eq. (3.12) is meaningful only in the context of NCDC. However, relations among the coefficient functions such as Eq. (3.2) 
hold irrespective of use of NCDC. As is assuring, the theorem (3.2) can also be proven [25] by a direct extension of the argument of Ref. [11].

\section{Proof of the lemmas}

In this section, we present the detailed proof of the lemmas in the preceding section.

\subsection{Algebraic Poincaré lemma}

To show Eq. (3.4), we start with the representation

$$
\eta(x)=\sum_{y} A_{\nu}(y) J_{\nu, y}(x)
$$

where the $p$-form $J_{\nu, y}(x)$ is given by ${ }^{\star}$

$$
J_{\nu, y}(x)=\int_{0}^{1} d t\left[\frac{\partial \eta(x)}{\partial A_{\nu}(y)}\right]_{A \rightarrow t A}
$$

and we have used the fact that $\left.\eta(x)\right|_{A=0}=0$. From Eq. (4.2), it is obvious that $d J_{\nu, y}(x)=0$ if $d \eta(x)=0$, and $\sum_{x} J_{\nu, y}(x)=0$ if $\sum_{x} \eta(x)=0$. Also the locality of $\eta(x)$ implies that $J_{\nu, y}(x)$ decreases at least exponentially as $|x-y| \rightarrow \infty$. Therefore the form $J_{\nu, y}(x)$ satisfies the requisite of the original Poincaré lemma (3.3). We thus have

$$
J_{\nu, y}(x)=d T_{\nu, y}(x)
$$

As noted in Eq. (3.10) below, the $(p-1)$-form $T_{\nu, y}(x)$ shares the same locality

\footnotetext{
$\star$ In the context of NCDC, the argument of a function changes depending on the order of form bases and the function. For this reason, the derivative with respect to $A_{\nu}(y)$ in this equation must be defined as the left derivative.
} 
property as $J_{\nu, y}(x)$. Then going back to Eq. (4.1), we have

$$
\begin{aligned}
\eta(x) & =d \sum_{y} A_{\nu}(y) T_{\nu, y}(x) \\
& =d \chi(x) .
\end{aligned}
$$

From the locality property of the $(p-1)$-form $T_{\nu, y}(x)$, it is obvious that the summation over $y$ is well-convergent and the $(p-1)$-form $\chi(x)$ is locally and smoothly depending on the gauge potential. We also note that $\left.\chi(x)\right|_{A=0}=0$. In this way, we have the algebraic Poincaré lemma, Eq. (3.4).

\subsection{Abelian BRST COHOMOlOgy}

The first step to show the BRST cohomology (3.5) is to choose a convenient set of bases in the functional space on the lattice, that is analogous to the jet variables in the continuum theory [17]. Noting the relations $f(x+\widehat{\mu})=\left(1+\partial_{\mu}\right) f(x)$ and $f(x-\widehat{\mu})=\left(1-\partial_{\mu}^{*}\right) f(x)$, we see that the gauge potential at an arbitrary point can be expressed as a linear combination of the terms with a structure

$$
\left(\partial_{1}\right)^{m_{1}}\left(\partial_{2}\right)^{m_{2}} \cdots\left(\partial_{D}\right)^{m_{D}} A_{\mu}(x)
$$

where $x$ is a certain reference point and $m_{\mu}$ 's are integer. In Eq. (4.5), we have introduced the notation

$$
\left(\partial_{\mu}\right)^{m}= \begin{cases}\partial_{\mu}^{m} & \text { for } m>0 \\ \partial_{\mu}^{*-m} & \text { for } m<0\end{cases}
$$

Note that, in Eq. (4.5), either the forward or the backward difference operator appears in each coordinate direction, but not both. Obviously the variables (4.5) with a different set of $m$ 's are linearly independent, because otherwise there exists a linear relation among the gauge potentials $A_{\mu}$ at different points. To discuss the BRST cohomology, however, it is convenient to separate gauge invariant combinations from the set of variables of Eq. (4.5). To do this, we note the following 
relations which "exchange" the order of indices of the difference operator and of the gauge potential

$$
\begin{aligned}
& \partial_{\mu} A_{\nu}(x)=\partial_{\nu} A_{\mu}(x)+F_{\mu \nu}(x) \\
& \partial_{\mu}^{*} A_{\nu}(x)=\left(1-\partial_{\mu}^{*}\right) \partial_{\nu} A_{\mu}(x)+\left(1-\partial_{\mu}^{*}\right) F_{\mu \nu}(x) .
\end{aligned}
$$

Namely, by a repeated use of these identities, we can always make the indices of the difference operators in Eq. (4.5) less or equal to that of the gauge potential $A_{\mu}$, up to terms linear in the field strength $F_{\mu \nu}$. After this manipulation, we can apply the identity

$$
\partial_{\mu}^{*} \partial_{\mu}=\partial_{\mu}-\partial_{\mu}^{*}
$$

to the product of the forward and the backward difference operators in the same direction, if necessary. In this way, the variable (4.5) can be expressed as a linear combination of

$$
A_{i}=\left(\partial_{1}\right)^{m_{1}} \cdots\left(\partial_{\mu}\right)^{m_{\mu}} A_{\mu}(x) \quad \text { and } \quad F_{i}=\left(\partial_{1}\right)^{m_{1}} \cdots\left(\partial_{D}\right)^{m_{D}} F_{\mu \nu}(x)
$$

It is also obvious that the variables $\left\{A_{i}\right\}$ and $\left\{F_{i}\right\}$ are linearly independent. For variables for the ghost field $c$, we choose

$$
c_{i}=\delta_{B} A_{i}=\left(\partial_{1}\right)^{m_{1}} \cdots\left(\partial_{\mu}\right)^{m_{\mu}} \partial_{\mu} c(x)
$$

Note that the combination of the difference operators is somewhat different from that of the representation (4.5), because Eq. (4.10) always contains at least one forward difference operator as $\cdots \partial_{\mu} c(x)$ and a single backward difference $\partial_{\mu}^{*} c(x)$ for example does not appear in Eq. (4.10). However the latter can be expressed as $\partial_{\mu}^{*} c=\partial_{\mu} c-\partial_{\mu}^{*} \partial_{\mu} c$ by the identity (4.8). The set of variables of Eq. (4.10) added with $c(x)$ is, therefore, equivalent to the choice $\left\{\left(\partial_{1}\right)^{m_{1}} \cdots\left(\partial_{D}\right)^{m_{D}} c(x)\right\}$. The variables $\left\{c_{i}\right\}$ and $c(x)$ span a complete set for the ghost field. 
With these preparations, we now turn to the proof of Eq. (3.5). We assume that the function $X$ in Eq. (3.5) is expressed in terms of the variables $\left\{A_{i}\right\},\left\{F_{i}\right\}$, $\left\{c_{i}\right\}$ and $c(x)$. To eliminate the ambiguity associated to the non-commutativity, we also assume that all the form bases are placed on the right of the field variables. We next note that the BRST transformation can be expressed in terms of these variables as

$$
\delta_{B}=\sum_{i} c_{i} \frac{\partial}{\partial A_{i}}
$$

because the variables $\left\{F_{i}\right\},\left\{c_{i}\right\}$ and $c(x)$ are BRST invariant. We also introduce the Grassmann-odd operator $r$

$$
r=\sum_{i} A_{i} \frac{\partial}{\partial c_{i}}
$$

Then the anti-commutator of $\delta_{B}$ and $r$ gives rise to the number operator of $\left\{A_{i}\right\}$ and $\left\{c_{i}\right\}$ :

$$
\left\{\delta_{B}, r\right\}=\sum_{i}\left(A_{i} \frac{\partial}{\partial A_{i}}+c_{i} \frac{\partial}{\partial c_{i}}\right)
$$

Finally, we introduce the auxiliary parameter $t$ in the function $X$ by rescaling the variables $\left\{A_{i}\right\}$ and $\left\{c_{i}\right\}$ as $A_{i} \rightarrow t A_{i}$ and $c_{i} \rightarrow t c_{i}$ (the variables $\left\{F_{i}\right\}$ and $c(x)$ are not rescaled). Then by writing $X_{t}=\left.X\right|_{A_{i} \rightarrow t A_{i}, c_{i} \rightarrow t c_{i}}$, we have

$$
\begin{aligned}
X & =X_{1}=X_{0}+\int_{0}^{1} d t \frac{\partial X_{t}}{\partial t} \\
& =X_{0}+\int_{0}^{1} \frac{d t}{t}\left\{\delta_{B}, r\right\} X_{t},
\end{aligned}
$$

where we have used Eq. (4.13). Since $\delta_{B}\left(t A_{i}\right)=t c_{i}$, if the function $X$ is BRST invariant $\delta_{B} X=0$, then the rescaled one $X_{t}$ is also BRST invariant $\delta_{B} X_{t}=0$. 
Therefore, from Eq. (4.14), we have

$$
\delta_{B} X=0 \Rightarrow X=X_{0}+\delta_{B} \int_{0}^{1} \frac{d t}{t} r X_{t},
$$

The first term $X_{0}$, which depends only on the gauge invariant variables $\left\{F_{i}\right\}$ and $c(x)$, is the BRST non-trivial piece (note that the BRST transformation of a certain function is proportional to $c_{i}$ ) and the second term gives the BRST exact piece. However, since $c(x)^{n}=0$ for $n \geq 2$ in Abelian gauge theories, we have the nontrivial piece only when the ghost number of the form $X$ is zero or one. This shows (after supplementing an appropriate $d \theta$ ) the Abelian BRST cohomology (3.5). The locality and the smoothness of the forms $\Omega$ and $Y$ in Eq. (3.5) are obvious from the representation (4.15) and the locality and the smoothness of the form $X$.

\subsection{Abelian covariant Poincaré lemma}

In the context of NCDC, the lemma (3.6) can be proven in an almost identical way as in the continuum theory [17], because we can use the Leibniz rule. To illustrate this point, we will emphasize how the Leibniz rule is used in the following proof. We show Eq. (3.6) by mathematical induction. For $p=0$, the lemma trivially holds by choosing $P=0$ because of the algebraic Poincaré lemma (3.4). Let us assume the lemma holds for $p=0,1, \cdots, n-1$. Now, for $n<D, \omega_{n}$ is $d$-closed and thus is $d$-exact by the algebraic Poincaré lemma. For $n=D$, it is $d$-exact by assumption. Also $\omega_{n}$ is $s$-closed because $\omega_{n}$ is gauge invariant:

$$
\omega_{n}=d \chi_{n-1}^{0}, \quad s \omega_{n}=0,
$$

where the upper index of $\chi$ stands for the ghost number and the lower index stands for the degree of the form. Note that the $(n-1)$-form $\chi_{n-1}^{0}$ is locally and smoothly depending on the gauge potential $A_{\mu}$ and $\left.\chi_{n-1}^{0}\right|_{A=0}=0$, as the result of the algebraic Poincaré lemma.

\footnotetext{
$\star$ The integral over the parameter $t$ is converging at $t=0$ because the possible $O\left(t^{0}\right)$ part of $X_{t}$ is eliminated by the derivative $\partial / \partial c_{i}$ in $r$.
} 
The two conditions (4.16) lead to the descent equation for the sequence of $\chi$. Namely, since $0=s \omega_{n}=s d \chi_{n-1}^{0}=--d s \chi_{n-1}^{0}$, the algebraic Poincaré lemma states that ${ }^{\dagger} s \chi_{n-1}^{0}=d \chi_{n-2}^{1}$. Similarly since $0=s^{2} \chi_{n-1}^{0}=s d \chi_{n-2}^{1}=-d s \chi_{n-2}^{1}$, we have $s \chi_{n-2}^{1}=d \chi_{n-3}^{2}$ again by the algebraic Poincaré lemma. Repeating this procedure, we have

$$
\begin{aligned}
s \chi_{n-1-g}^{g} & =d \chi_{n-2-g}^{g+1}, \quad 0 \leq g<n-1, \\
s \chi_{0}^{n-1} & =0 .
\end{aligned}
$$

Note that all $\chi$ 's are locally and smoothly depending on the gauge potential and on the ghost field and that $\left.\chi\right|_{A=c=0}=0$ by the algebraic Poincaré lemma.

Now the solution of the last equation in Eq. (4.17) with $n \neq 1$ is given by the Abelian BRST cohomology (3.5),

$$
\chi_{0}^{n-1}= \begin{cases}C \Omega_{0}+s b_{0}, & \text { for } n=2, \\ s b_{0}, & \text { otherwise }\end{cases}
$$

where the 0 -form $\Omega_{0}$ is gauge invariant and $\Omega_{0}$ and $b_{0}$ are locally and smoothly depending on the gauge potential and on the ghost field. But by redefining $\chi_{0}^{n-1}$ and $\chi_{1}^{n-2}$ as

$$
\chi_{0}^{n-1} \rightarrow \chi_{0}^{n-1}+s b_{0}, \quad \chi_{1}^{n-2} \rightarrow \chi_{1}^{n-2}-d b_{0},
$$

we can completely eliminate $b_{0}$-dependences from the descent equation (4.17). Note that this redefinition preserves the property $\left.\chi\right|_{A=c=0}=0$, because $s b_{0}$ and $d b_{0}$ cannot be a constant. Without loss of generality, therefore, we can take

$$
\chi_{0}^{n-1}= \begin{cases}C \Omega_{0}, & \text { for } n=2, \\ 0, & \text { otherwise. }\end{cases}
$$

Repeating this argument in Eq. (4.17), we see that the non-trivial part of the

$\dagger$ The algebraic Poincaré lemma in its original form (3.4) applies to a form that depends only on the gauge potential $A_{\mu}$. However it is straightforward to generalize the lemma for forms which contain the ghost field $c$ as well. The property $\left.\chi(x)\right|_{A=0}=0$ is then replaced by $\left.\chi(x)\right|_{A=c=0}=0$. 
descent equation is given by

$$
\begin{aligned}
& s \chi_{n-1}^{0}=d \chi_{n-2}^{1}, \\
& s \chi_{n-2}^{1}=0 .
\end{aligned}
$$

The solution of the last equation in Eq. (4.21) can be taken as $\chi_{n-2}^{1}=C \Omega_{n-2}=$ $C\left(B_{n-2}+\omega_{n-2}\right)$, here $B_{n-2}$ stands for the constant part of $\Omega_{n-2},\left.\Omega_{n-2}\right|_{A=0}=B_{n-2}$ and $\left.\omega_{n-2}\right|_{A=0}=0$. The form $\omega_{n-2}$ is locally and smoothly depending on the gauge potential $A_{\mu}$ and is gauge invariant. The first relation in Eq. (4.21) then yields

$$
\begin{aligned}
s \chi_{n-1}^{0} & =d\left[C\left(B_{n-2}+\omega_{n-2}\right)\right] \\
& =d C\left(B_{n-2}+\omega_{n-2}\right)-C d \omega_{n-2} \\
& =-s A\left(B_{n-2}+\omega_{n-2}\right)-C d \omega_{n-2},
\end{aligned}
$$

where we have used the Leibniz rule and Eq. (2.15). Now the relation (4.22) holds for arbitrary configurations of the ghost field $c(x)$. When the ghost field is a constant $c(x)=c$, Eq. (4.22) reduces to $C d \omega_{n-2}(x)=0$ because other terms in the equation are proportional to the difference of the ghost field $\partial_{\mu} c(x)=c(x+\widehat{\mu})-c(x)$ etc. Therefore we see that the $(n-2)$-form $\omega_{n-2}$ must be $d$-closed and

$$
s\left[\chi_{n-1}^{0}+A\left(B_{n-2}+\omega_{n-2}\right)\right]=0, \quad d \omega_{n-2}=0 .
$$

The solution of the first equation in Eq. (4.23) is given by the BRST cohomology (3.5),

$$
\chi_{n-1}^{0}=-A\left(B_{n-2}+\omega_{n-2}\right)+\Omega_{n-1} .
$$

Note that the $(n-1)$-form $\Omega_{n-1}$ is gauge invariant and again is locally and smoothly depending on $A_{\mu}$ and $\left.\Omega_{n-1}\right|_{A=0}=0$. 
Finally, substituting Eq. (4.24) in Eq. (4.16), we have

$$
\begin{aligned}
\omega_{n} & =d\left[-A\left(B_{n-2}+\omega_{n-2}\right)+\Omega_{n-1}\right] \\
& =-F\left(B_{n-2}+\omega_{n-2}\right)+A d \omega_{n-2}+d \Omega_{n-1} \\
& =-F\left(B_{n-2}+\omega_{n-2}\right)+d \Omega_{n-1} .
\end{aligned}
$$

Here we have used the Leibniz rule and the fact that the form $\omega_{n-2}$ is $d$-closed $d \omega_{n-2}=0$, as is shown in Eq. (4.23). However, by the induction hypothesis, the lemma (3.6) applies to the gauge invariant closed $(n-2)$-form $\omega_{n-2}$ (recall that $\left.\left.\omega_{n-2}\right|_{A=0}=0\right)$ :

$$
\omega_{n-2}=P(F)+d \eta
$$

Equations (4.25) and (4.26) then show that the lemma (3.6) holds for $\omega_{n}$ also:

$$
\begin{aligned}
\omega_{n} & =-F\left[B_{n-2}+P(F)\right]-F d \eta+d \Omega_{n-1} \\
& =-F\left[B_{n-2}+P(F)\right]+d\left(-F \eta+\Omega_{n-1}\right),
\end{aligned}
$$

where uses of the Leibniz rule and the Bianchi identity $d F=0$ have been made. Note that all the coefficients in $P(F)$ appear on the right of $F$ 's in the iteration of Eqs. (4.26) and (4.27). The locality and the smoothness of the gauge invariant quantity in the round brackets in the second equation in Eq. (4.27) are obvious from the above argument. In this way, we establish the lemma Eq. (3.6).

\section{Conclusion}

In this paper, we have presented an algebraic proof of the theorem (1.3) and its generalization to arbitrary dimensions (3.2) on the basis of cohomological arguments. The basic tool in our approach is NCDC which makes the Leibniz rule of exterior derivatives valid on a lattice. By using NCDC, we can readily transcribe the proof of the corresponding statement in the continuum theory, such as the (Abelian) linearized covariant Poincaré lemma [17], into the language of lattice 
theory. We can also easily understand the topological nature of the Chern character on a lattice. In these respects, our algebraic approach is complementary to the constructive proof in Ref. [11].

The explicit form of the gauge invariant local current $k_{\mu}(x)$ in Eqs. (1.3) and (3.2) is of great interest because it is a crucial ingredient in the Abelian lattice chiral gauge theories formulated in Ref. [15]. For example, the gauge invariant effective action on an infinite lattice is given by a simple integral representation, once the explicit form of the current $k_{\mu}(x)$ is known [30]. From Eq. (3.12), we see that the current $k_{\mu}(x)$ or equivalently the form $\eta(x)$ can be constructed by applying the algebraic Poincaré lemma (3.4) to $[q(x)-\alpha] d^{D} x-P(F(x))$. From the proof of the algebraic Poincaré lemma in Sec. 4.1 (and from the proof of the original Poincaré lemma [11]), this implies that the current $k_{\mu}(x)$ is given by a linear combination of a product of the gauge potential $A_{\mu}$ and the first derivative of $q(x)$ with respect to $A_{\mu}$. Unfortunately, however, the resulting expression seems to be too complicated to be of any practical use.

As a simple application of our theorem (3.2), we may consider the general gauge invariant solution of $\sum_{x} \int d t d s \delta q(z)=0$ in Abelian gauge theories in the $(4+2)$ dimensional space, here 4-dimensions are discrete (with coordinates specified by $x$ ) and the remaining 2-dimensions are continuous (their coordinates are $t$ and $s$ ). This is the cohomological problem posed in Ref. [26], in connection with the gauge invariant formulation of lattice chiral gauge theories. When the gauge group is Abelian, the general solution may simply be obtained by taking the classical continuum limit of Eq. (3.2) with respect to the continuous 2-dimensional space. Then the topological quantity $q(z)$, up to terms irrelevant for the discussions in Ref. [26], is given by

$$
\begin{aligned}
q(z)= & \gamma \varepsilon_{I J K L M N} F_{I J}(x, t, s) F_{K L}(x+\widehat{I}+\widehat{J}, t, s) F_{M N}(x+\widehat{I}+\widehat{J}+\widehat{K}+\widehat{L}, t, s) \\
& +\partial_{\mu}^{*} k_{\mu}(z)+\partial_{t} k_{t}(z)+\partial_{s} k_{s}(z)
\end{aligned}
$$

where the Latin indices run from 1 to 6 and the Greek indices run over only 
from 1 to 4 ; the vectors along the continuous directions are zero $\widehat{5}=\widehat{6}=0$. For the topological quantity $q(z)$ which are relevant for the analysis in Ref. [26], the coefficient $\gamma$ is proportional to a sum of the cubic of $U(1)$ charges of Weyl fermions, i.e., the gauge anomaly. Therefore, when the $U(1)$ gauge anomaly cancellation condition is fulfilled, the quantity $q(z)$ is given by total divergences. The discussion of Ref. [26] then shows that it is possible to formulate Abelian gauge theories on the lattice, while keeping the exact gauge invariance. Of course this should be so from the result obtained in Ref. [15], but the present example clearly illustrates the usefulness of our result (3.2) for a higher-dimensional lattice.

We are deeply indebted to M. Lüscher for helpful comments on the previous versions of this paper. We are grateful to F. Müller-Hoissen for information and to K. Fujikawa for helpful comments. K.W. is very grateful to F. Sakata for the warm hospitality extended to him and to Faculty of Science of Ibaraki University for the financial support during his visit at Ibaraki University.

\section{Note added:}

The statement made in Eq. (4.9) below that the variables $\left\{F_{i}\right\}$ are linearly independent is not correct. The Bianchi identity and its differences are linear relations among $F_{i}$ 's. However, the proof of the BRST cohomology does not change if we assume that the function $X$ is expressed in terms of $\left\{A_{i}\right\},\left\{c_{i}\right\}, c(x)$, and the independent set of variables in $\left\{F_{i}\right\}$. 


\section{REFERENCES}

1. P. H. Ginsparg and K. G. Wilson, Phys. Rev. D25 (1982) 2649.

2. P. Hasenfratz, Nucl. Phys. (Proc. Suppl.) 63 (1998) 53; Nucl. Phys. B525 (1998) 401.

3. P. Hasenfratz, V. Laliena and F. Niedermayer, Phys. Lett. B427 (1998) 125.

4. H. Neuberger, Phys. Lett. B417 (1998) 141; B427 (1998) 353.

5. M. Lüscher, Phys. Lett. B428 (1998) 342.

6. K. Fujikawa, Phys. Rev. Lett. 42 (1979) 1195; Phys. Rev. D21 (1980) 2848; D22 (1980) 1499 (E); D29 (1984) 285.

7. Y. Kikukawa and A. Yamada, Phys. Lett. B448 (1999) 265.

8. D. H. Adams, hep-lat/9812003.

H. Suzuki, Prog. Theor. Phys. 102 (1999) 141.

9. K. Fujikawa, Nucl. Phys. B546 (1999) 480.

10. T.-W. Chiu, Phys. Lett. B445 (1999) 371.

T.-W. Chiu and T.-H. Hsieh, hep-lat/9901011.

T. Reisz and H. J. Rothe, Phys. Lett. B455 (1999) 246.

11. M. Lüscher, Nucl. Phys. B538 (1999) 515.

12. R. Narayanan, Phys. Rev. D58 (1998) 097501.

F. Niedermayer, Nucl. Phys. (Proc. Suppl.) 73 (1999) 105.

Y. Kikukawa and A. Yamada, Nucl. Phys. B547 (1999) 413.

13. H. Neuberger, Phys. Rev. D59 (1999) 085006.

14. R. Narayanan and H. Neuberger, Nucl. Phys. B412 (1994) 574; B443 (1995) 305.

S. Randjbar-Daemi and J. Strathdee, Phys. Lett. B402 (1997) 134.

15. M. Lüscher, Nucl. Phys. B549 (1999) 295. 
16. K. Fujikawa, Phys. Rev. D60 (1999) 074505.

T. Aoyama and Y. Kikukawa, hep-lat/9905003.

T.-W. Chiu, hep-lat/9906007.

17. F. Brandt, N. Dragon and M. Kreuzer, Phys. Lett. B231 (1989) 263; Nucl. Phys. B332 (1990) 224; B332 (1990) 250.

N. Dragon, Lectures given at Saalburg Summer School (1995), hep-th/9602163.

18. M. Dubois-Violette, M. Henneaux, M. Talon and C.-M. Viallet, Phys. Lett. B267 (1991) 81; B289 (1992) 361.

19. B. Zumino, Y. S. Wu and A. Zee, Nucl. Phys. B239 (1984) 477.

B. Zumino, in Relativity, Groups and Topology II, eds. B. S. De Witt and R. Stora, (North-Holland, Amsterdam, 1984).

L. Baulieu, Nucl. Phys. B241 (1984) 557; in Progress in Gauge Field Theory, eds. H. Lehmann et. al., NATO ASI Series B, Physics, Vol. 115 (Plenum, New York, 1984).

R. Stora, in Progress in Gauge Field Theory, eds. H. Lehmann et. al., NATO ASI Series B, Physics, Vol. 115 (Plenum, New York, 1984).

20. A. Connes, Noncommutative Geometry, (Academic Press, New York, 1994).

21. A. Sitarz, J. Geom. Phys. 15 (1995) 123.

22. A. Dimakis and F. Müller-Hoissen, Phys. Lett. B295 (1992) 242; J. Phys. A: Math. Gen. 27 (1994) 3159; J. Math. Phys. 35 (1994) 6703.

A. Dimakis, F. Müller-Hoissen and T. Striker, Phys. Lett. B300 (1993) 141;

J. Phys. A: Math. Gen. 26 (1993) 1927.

23. H. G. Ding, H. Y. Guo, J. M. Li and K. Wu, Z. Phys. C64 (1994) 521; J. Phys. A: Math. Gen. 27 (1994) L75; 27 (1994) L231; Commun. Theor. Phys. 21 (1994) 85.

H. Y. Guo, K. Wu and W. Zhang, "Noncommutative Differential Calculus on Discrete Abelian Groups and Its Applications," ITP-Preprint, March, 1999. 
24. A. P. Balachandran, G. Bimonte, G. Landi, F. Lizzi and P. TeotonioSobrinho, J. Geom. Phys. 24 (1998) 353, and references therein.

25. T. Fujiwara, H. Suzuki and K. Wu, Phys. Lett. B463 (1999) 63.

26. M. Lüscher, hep-lat/9904009.

27. I. Horvath, Phys. Rev. Lett. 81 (1998) 4063; Phys. Rev. D60 (1999) 034510. W. Bietenholz, hep-lat/9901005.

28. P. Hernández, K. Jansen and M. Lüscher, Nucl. Phys. B552 (1999) 363.

29. A. Dimakis and F. Müller-Hoissen, physics/9712004.

30. H. Suzuki, Prog. Theor. Phys. 101 (1999) 1147. 